NASA Technical Memorandum 106241

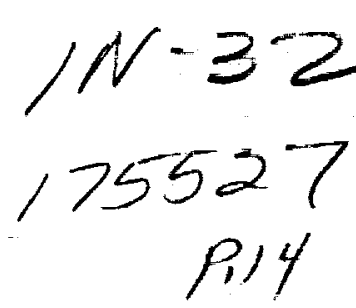

\title{
Combinatorial Pulse Position Modulation for Power-Efficient Free-Space Laser Communications
}

James M. Budinger

National Aeronautics and Space Administration

Lewis Research Center

Cleveland, Ohio

M. Vanderaar and P. Wagner

Sverdrup Technology, Inc.

Lewis Research Center Group

Cleveland, Ohio

and

Steven Bibyk

Ohio State University

Columbus, Ohio

Prepared for the

Society of Photo-Optical Instrumentation Engineers

sponsored by the International Society for Optical Engineering

Los Angeles, California, January 16-23, 1993 


\title{
COMBINATORIAL PULSE POSITION MODULATION FOR POWER-EFFICIENT
}

\section{FREE-SPACE LASER COMMUNICATIONS}

\author{
James M. Budinger \\ National Aeronautics and Space Administration \\ Lewis Research Center \\ Cleveland, Ohio 44135
}

\author{
M. Vanderaar and P. Wagner \\ Sverdrup Technology, Inc. \\ Lewis Research Center Group \\ Brook Park, Ohio 44142
}

and

\author{
Steven Bibyk \\ Ohio State University \\ Columbus, Ohio 44321
}

\begin{abstract}
A new modulation technique called combinatorial pulse position modulation (CPPM) is presented as a power-efficient altemative to quaternary pulse position modulation (QPPM) for direct-detection, free-space laser communications. The special case of ${ }_{16} C_{4} \mathrm{PPM}$ is compared to QPPM in terms of data throughput and bit error rate (BER) performance for similar laser power and pulse duty cycle requirements. The increased throughput from CPPM enables the use of forward error corrective (FEC) encoding for a net decrease in the amount of laser power required for a given data throughput compared to uncoded QPPM. A specific, practical case of coded CPPM is shown to reduce the amount of power required to transmit and receive a given data sequence by at least $4.7 \mathrm{~dB}$. Novel hardware techniques for maximum likelihood detection and symbol timing recovery are presented.

\section{INTRODUCTION}

Pulse position modulation (PPM) is a technique suitable for direct detection of an optical signal transmitued through space. Each pulse of a laser can be used to represent one or more bits of information by its position in time relative to the start of a symbol. For example, in binary pulse position modulation, also known as Manchester encoding, a single bit of information is represented by a symbol with a pulse in one of two distinct positions, or slots, within the symbol. The symbol duration is identical to that of the information bit. A logical zero could be conveyed by a pulse present in the first slot, whereas a pulse in the second slot could represent a logical one. Quatemary pulse position modulation (QPPM) encodes two bits of information into each four-slot symbol. Again, only a single pulse of the laser occupies the symbol, which is now identical in duration to two bit periods. In like fashion, $M$-ary PPM extends the number of bits represented by each of $M$ distinct symbols to $\log _{2} M$. In theory, 256-PPM or higher $M$-ary PPM techniques could be used to convey eight or more bits of information at a time. In practice, physical constraints of producing and detecting narrower and narrower pulses with the same energy content, and difficulties in recovering the symbol timing reference, limit their use in high data rate applications through free space.

The National Aeronautics and Space Administration (NASA) has proposed the use of direct detection optical communications as an alternative to conventional radio frequency/microwave links for power limited intersatellite and deepspace applications. $1,2,3$ NASA has proposed the use of QPPM in high data rate (to $650 \mathrm{Mbps}$ ) communications links between geostationary satellites. The selection of QPPM over higher $M$-ary techniques is governed primarily by the availability of wideband, high-sensitivity, avalanche photodiodes (APDs), and the difficulty of recovering symbol clock timing in higher $M$ ary techniques. QPPM also reduces the laser duty cycle by a factor of two compared to binary PPM while preserving a peakto-average power ratio that does not appreciably decrease the life of the laser diode. Under work sponsored by NASA Goddard Space Flight Center, Davidson and Sun have developed techniques for synchronizing clocks and recovering QPPM modulated data at rates to 220 Mbps. ${ }^{4,5}$ At NASA Lewis Research Center, the electronics for dual-channel, full duplex QPPM at 325 Mbps per channel are under development ${ }^{6}$ To alleviate the constraint of producing and detecting narrower and narrower optical pulses to convey more information for a given amount of available power, techniques such as trellis coding of overlapped PPM have been investigated. ${ }^{7}$ The technique developed in this paper, combinatorial pulse position modulation (CPPM), addresses power efficient laser communications as well.
\end{abstract}


In conventional $M$-ary PPM, the product of different symbols over one symbol period is zero. This property defines $M$-ary PPM as an orthogonal signalling scheme. While orthogonal signalling provides improved power efficient communications with increasing $M$, its increased peak-to-average power ratio makes it problematic for laser diode based communications. $M$-ary PPM is a subset of CPPM in which only one pulse per symbol period is allowed. In CPPM, a symbol can contain more than one pulse. The number of valid symbols in the alphabet could be as large as the number of combinations of $a$ things (slots) taken $b$ (pulses) at a time $\left({ }_{a} C_{b}\right)$. For example, in ${ }_{12} C_{2}$ PPM there are 66 combinations or unique symbols, each consisting of 12 slots filled by 2 pulses per symbol. These 66 symbols are more than enough to convey 6 bits of information per symbol since only a $2^{6}=64$ symbol alphabet is required. QPPM would require one additional pulse over 12 slots to convey the same 6 bits of information. 64-PPM would require laser pulses with over 10 times the peak-toaverage power ratio and require electronics with over 5 times the bandwidth.

There are several practical issues to be addressed and resolved before CPPM should be considered as a viable alternative to QPPM or higher $M$-ary PPM techniques: (1) the selection of an efficient alphabet considering laser diode constraints and realistic synchronization mechanisms; (2) the bit-error-rate versus signal-to-noise performance compared to other PPM techniques; (3) the use of FEC coding available from the increased throughput; and (4) the implementation approach for symbol timing recovery and maximum likelihood data detection. These topics are discussed in the sections that follow. Throughout the paper, an emphasis on practical implementation drives the selection of approaches to CPPM. For direct comparison to the 325 Mbps QPPM hardware under development at NASA, only CPPM techniques that place nearly identical constraints on the laser transmitter and APD receiver are considered. Within these constraints, we will show that a specific practical case of coded CPPM reduces the amount of power required to transmit and receive the same digital data sequence by at least $4.7 \mathrm{~dB}$. We will also describe practical techniques for implementing the symbol clock and data recovery.

\section{COMBINATORIAL PPM THEORY}

In the late 1960 's, Slepian ${ }^{8}$ described a general class of "group codes for the bandlimited channel". These codes were later considered for use in conjunction with frequency shift keyed (FSK) modulation $9,10,11$. These schemes have been generally referred to as permutation modulation. The application of permutation modulation to PPM is similar to that in combinatorial frequency shift keying (CFSK) ${ }^{12}$, except that time slot selection takes the place of frequency bin selection. A CPPM modulator transforms data into symbols by populating $b$ out of $a$ available time slots with equal-energy pulses. A CPPM demodulator identifies the $b$ highest energy time slots in each symbol and declares the data bits defined by that symbol.

The size of a general CPPM alphabet is

$$
\mathrm{a}_{\mathrm{b}}=\frac{\mathrm{a} !}{\mathrm{b} !(\mathrm{a}-\mathrm{b}) !}
$$

symbols. For example, a general CPPM alphabet with 16 slots and 4 pulses per symbol contains ${ }_{16} C_{4}=1820$ unique symbols and is thus capable of conveying $\left[\log _{2} 1820\right\rfloor=10$ information bits per symbol. The residual capacity ( 1820 . 1024 = 796 symbols ) can be exploited to improve CPPM system operation in two ways. First, by forcing the last slot in each symbol to be unpopulated, strong spectral lines at integer multiples of the symbol rate are created, thus providing for robust symbol timing recovery in the CPPM demodulator. This results in an alphabet size of $15 C_{4}=1365$ symbols. Second, by discarding symbols with 3 or 4 adjacent slots populated, the maximum on time duration can be limited to 2 slots, just as in QPPM. Pulse width and duty cycle are important considerations in solid-state laser transmitter design and reliability. With both improvements in place, the alphabet size is reduced to 1221 symbols, still supporting a 10 bit per symbol throughput capacity.

By removing an additional 197 symbols from the general ${ }_{16} C_{4}$ alphabet, exactly $2^{10}=1024$ symbols remain to allow a one-to-one mapping between CPPM symbols and 10-bit data words. The CPPM system which has the special reduced alphabet described above shall be referred to as ${ }_{16} C_{4} \mathrm{PPM}$. The motivation to study the performance of ${ }_{16} C_{4} \mathrm{PPM}$ versus QPPM is based on the observation that their peak-to-average power ratios and average duty cycles are equal, yet ${ }_{16} C_{4} \mathrm{PPM}$ has a 25\% higher data throughput capacity. 
The question arises as to what choice of data to symbol mapping would be appropriate. For this study, the 102410 bit data words were sorted in ascending order of Hamming distance. The ${ }_{16} \mathrm{C}_{4} \mathrm{PPM}$ symbols with the 1024 highest Hamming distances were then assigned to entries in the sorted 10-bit data word list. The remaining 197 symbols were discarded and are treated as invalid inputs when received by the ${ }_{16} C_{4}$ PPM demodulator. By taking advantage of distance properties in this manner, this mapping technique provides modest performance advantages over a simple random alphabet assignment. A more significant performance advantage based on distance structure would require a much larger signal space than is available in ${ }_{16} C_{4} \mathrm{PPM}$.

\section{PERFORMANCE OF OPPM AND CPPM}

A meaningful performance measurement of a digital modulation scheme is the bit-error-rate (BER) as a function of signal-to-noise ratio. When this indicator of power efficiency is normalized for data throughput, various modulation schemes can be compared in a fair manner. The BER performance of $M$-ary PPM in additive white Gaussian noise (AWGN) can be upper bounded by the expression for $M$-ary orthogonal signalling ${ }^{13}$ :

$$
P_{b}(m) \leq \frac{2^{m-1}}{2} \operatorname{erf}\left(\sqrt{\frac{m E_{b}}{2 N_{0}}}\right)
$$

where for QPPM, the number of bits per symbol, $m=\log _{2} M=\log _{2} 4=2$. The BER vs. $E_{b} / N_{o}$ result for QPPM is plotted in Figure 1. The use of the Gaussian noise approximation in the calculation of system bit error probabilities has been shown to be accurate in situations of low background radiation and low avalanche photodiode bulk leakage current ${ }^{14}$.

A simple analytical determination of the performance of ${ }_{16} C_{4} \mathrm{PPM}$ results in bounds that are not tight enough for accurate performance evaluations. For this reason, time domain digital simulations were conducted for both QPPM and ${ }_{16} C_{4}$ PPM using the Signal Processing Worksystem ${ }^{T M}$ (SPW) ${ }^{15}$. Equivalent block diagrams of the system are shown in Figures 2 and 3 . The simulations measure a slot error rate which is then converted to an equivalent symbol error rate. The symbol-to-noise ratio, $E_{s} / N_{O}$, can be adjusted in accordance with the finite extinction ratio (FER) characteristics of the modulated laser diode. For example, a FER of $10 \%$ has the effect of putting $40 \%$ more noise power into each symbol (CPPM or QPPM) without decreasing the error probabilities. The net result of a 10\% FER is a $2.22 \mathrm{~dB}$ loss in both QPPM and CPPM performance. For a $5 \%$ FER the loss is $0.97 \mathrm{~dB}$. In the CPPM case, the slot error rate is converted into a CPPM symbol error rate by the conservative upper bound assumption that two slot errors result in one symbol error. This bound is exact for the case in which two slot errors occur per CPPM symbol. The symbol error rate is converted to the bit error rate by the following expression

$$
P_{b}=\frac{2^{m-1}}{2^{m}-1} P_{s}
$$

where $P_{s}$ is the symbol error rate, again $m$ is the number of bits per PPM symbol, and $P_{b}$ is the bit error rate. This expression is valid for the random alphabet assignment, which is a good approximation in the ${ }_{16} C_{4} \mathrm{PPM}$ case. For each simulation result at least 100 error events were recorded to give a statistically accurate estimate of the bit error probability. These results are also ploted in Figure 1 and are equalized for throughput, enabling a fair comparison between the schemes. It is seen that the upper bound for QPPM is progressively tight for increasing $E_{b} / N_{o}$. Further, the performance of ${ }_{16} C_{4} \mathrm{PPM}$ is approximately $I d B$ better than that of QPPM. 

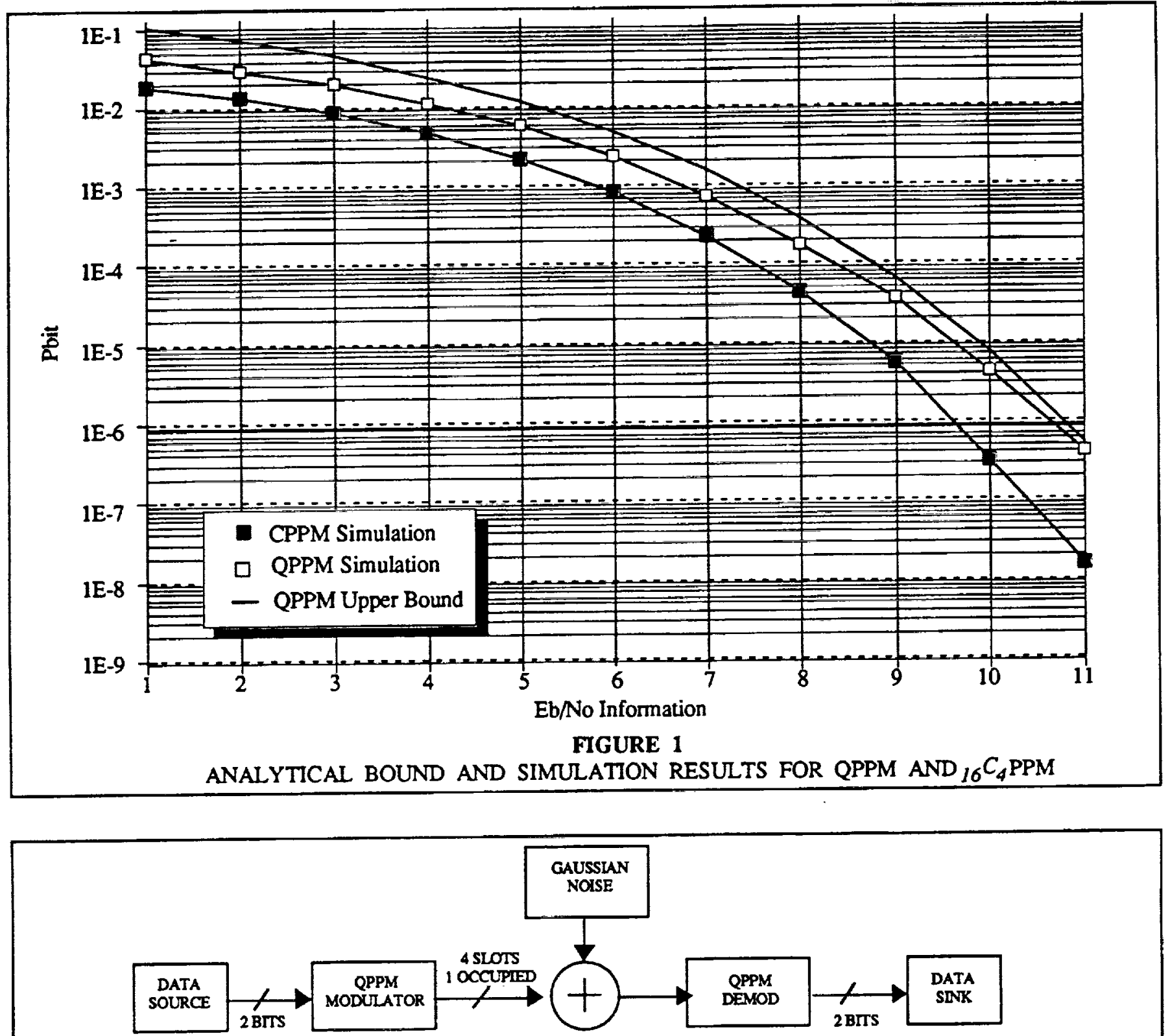

FIGURE 2

SIMULATION MODEL FOR QPPM

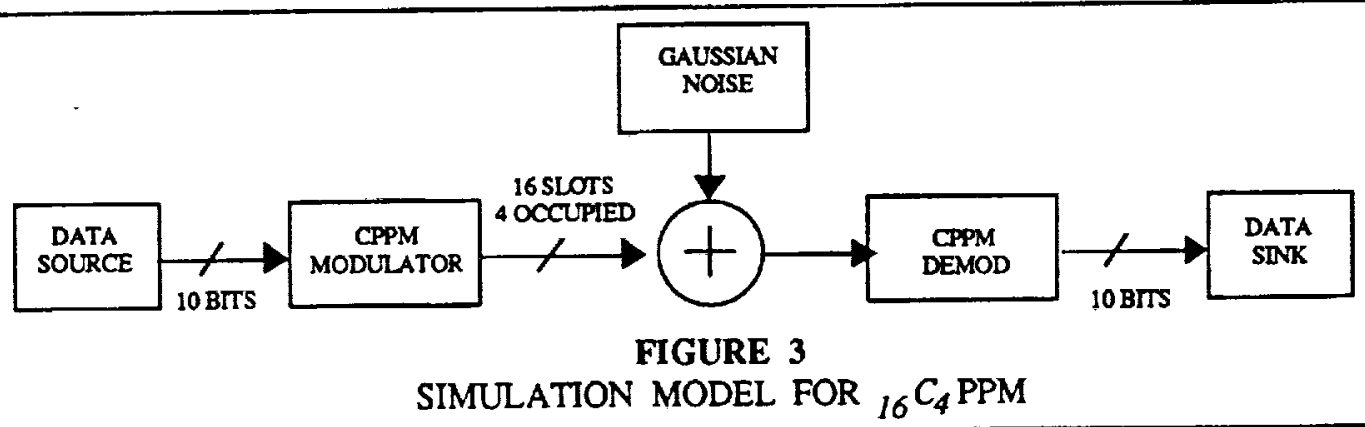




\section{BLOCK CODING FOR CPPM}

In addition to the performance gains obtained by CPPM through the expansion of the modulation set, further improvements are possible through the use of FEC codes. In the case of ${ }_{16} C_{4} \mathrm{PPM}$ the $25 \%$ increase in throughput over QPPM (or equivalent $I d B$ increase in power efficiency) can be used as redundancy in an FEC code to increase the power efficiency over QPPM while maintaining the same throughput at 0.5 information bits per PPM slot. One method of applying forward error corrective (FEC) coding to a CPPM scheme is to encode the information symbols with a rate $k / n$ code, where $n / k$ is equivalent to the throughput ratio of the CPPM scheme to the orthogonal PPM scheme. This study limits its scope to the class of FEC codes called block codes. The motivation for this is due to the relative ease of implementing block decoders at high data rates. Additionally, as will be shown, block codes are well matched to the CPPM modulation scheme.

Over the same 16 slots, the throughput gain of ${ }_{16} C_{4} \mathrm{PPM}$ over QPPM is 10 bits to 8 bits. This allows for a rate $8 / 10=4 / 5$ code that precedes the bit to symbol mapping function. The approach to coding ${ }_{16} C_{4} \mathrm{PPM}$ should be matched to the error characteristics of the uncoded scheme. Since alphabet selection based on Hamming distance does not allow significant enror performance gains, a random 10 bits to 1 CPPM symbol mapping will be assumed. The specific probability that $r$ out of $v$ bits will be in error given a symbol error is determined as

$$
P_{r}=\frac{\left(\begin{array}{l}
v \\
r
\end{array}\right)}{\sum_{i=1}^{v}\left(\begin{array}{l}
v \\
i
\end{array}\right)}=\frac{\left(\begin{array}{l}
v \\
r
\end{array}\right)}{2^{v}}
$$

Table 1 shows the probability that a symbol error was caused by $r$ bit errors. This indicates that the performance of a scheme that uses anything less than an $r$ bit error correcting code will be dominated by events with $r+1$ or more bit errors. Due to the difficulty in constructing such bit error correcting codes at high data rates, block codes defined over an extension field (in this case $G F\left(2^{10}\right)$ ) that are matched to the symbol size are a more appropriate choice for protecting ${ }_{16} C_{4} \mathrm{PPM}$ modulated data. One candidate rate $8 / 10$ code that results in an equal throughput to QPPM is a Reed-Solomon $(n, k, t)$ code with a total length $n=102410$ bit symbols and $k=820$ information characters, correcting $t=102$ characters. This can be denoted as a $R S(1024,820,102)$ code. Each ${ }_{16} C_{4} \mathrm{PPM}$ symbol and Reed-Solomon character are both 10 bits, thus matching the code to the modulation. While this code would offer excellent performance, its length and the length of its syndrome make it impractical for implementation. For this reason, Reed-Solomon codes based on the smaller extension fields $G F\left(2^{l}\right), k 10$ are more applicable for this system.

TABLE 1

PROBABILITY OF ERRORS OF HAMMING WEIGHT $r$ for $v=10$

\begin{tabular}{lllllll}
\hline \hline$r$ & 0,10 & 1,9 & 2,8 & 3,7 & 4,6 & 5 \\
\hline$P_{r}$ & $9.77 \mathrm{E}-5$ & $9.77 \mathrm{E}-4$ & $4.39 \mathrm{E}-2$ & $1.17 \mathrm{E}-1$ & $2.05 \mathrm{E}-1$ & $2.46 \mathrm{E}-1$
\end{tabular}

Based on the error correcting capability of commercially available Reed-Solomon codec chip sets, the longest practical rate 8/10 RS code selected for investigation was defined over $G F\left(2^{8}\right)$. The parameters and the performance of four codes applied to ${ }_{16} C_{4}$ PPM are listed in Table 2 and illustrated in Figure 4. These results were determined through a combination of simulation and analysis. First, the simulation results for uncoded ${ }_{16} C_{4} \mathrm{PPM}$ are used to determine the input character error rate for the Reed-Solomon decoder for the coded CPPM system depicted in Figure 5. Since this uncoded performance data was gathered from a time domain simulation with a minimum of approximations, it is more accurate than an analytical bound, especially at low $E_{b} / N_{o}$. The accuracy of uncoded low $E_{b} / N_{o}$ data is important when evaluating the performance of ReedSolomon coding schemes due to the dramatic sensitivity of decoded error rate to minor changes in the input error rate. An upper bound on the error rate can be determined by assuming that each ${ }_{16} C_{4}$ PPM symbol error causes exactly two Reed-Solomon character errors. For example, when the $R S(32,26,3)$ code that is based on 5 bit characters encounters a ${ }_{16} C_{4}$ PPM symbol error ( 10 bits in length) it is assumed that 2 characters will be in error. This bound becomes looser as the Reed-Solomon characters approach 10 bits in length. In the 10 bit limit it is clear that one ${ }_{16} C_{4}$ PPM symbol error can cause only one Reed- 
Solomon character error. With a t-error correcting decoder that makes a maximum of $t+x$ output character ertors when $x>t+1$ input character errors occur, an upper bound on the decoded character error probability can be written as

$$
P_{c} \leq \sum_{\substack{x=t+1 \\
x \\
\text { even }}}^{n} \frac{x+1}{n}\left(\begin{array}{l}
n \\
x
\end{array}\right)\left(P_{s C P P M}\right)^{x / 2}\left(1-P_{s C P P M}\right)(n-x) / 2
$$

This decoded character error rate can then be converted to a decoded bit error rate by equation 3 .

TABLE 2

REED-SOLOMON CODE PARAMETERS AND UPPER BOUND PERFORMANCE

\begin{tabular}{lcccccc}
\hline \hline $\mathbf{n}$ & $\mathbf{k}$ & $\mathrm{t}$ & $\begin{array}{c}\text { bits per } \\
\text { character }\end{array}$ & $\begin{array}{c}\text { rate } \\
(\mathrm{k} / \mathrm{n})\end{array}$ & $\begin{array}{c}\text { rate loss } \\
(\mathrm{dB})\end{array}$ & $\begin{array}{c}\text { coding gain } \\
@ \mathrm{~Pb}=10 \mathrm{e}-6\end{array}$ \\
\hline uncoded & $\mathrm{N} / \mathrm{A}$ & 0 & $\mathrm{~N} / \mathrm{A}$ & $\mathbf{l}$ & 0.0000 & $0 \mathrm{~dB}$ \\
32 & 26 & 3 & 5 & 0.8125 & 0.9018 & $0.8 \mathrm{~dB}$ \\
64 & 50 & 7 & 6 & 0.78125 & 1.0721 & $2.7 \mathrm{~dB}$ \\
128 & 102 & 13 & 7 & 0.796875 & 0.986 & $3.9 \mathrm{~dB}$ \\
256 & 206 & 25 & 8 & 0.8046875 & 0.943 & $4.7 \mathrm{~dB}$ \\
\hline
\end{tabular}

* Note that the coding gains are measured as compared to uncoded ${ }_{16} C_{4} \mathrm{PPM}$ and are normalized for equal throughput. Add a gain of approximately $1 \mathrm{~dB}$ for comparison to uncoded QPPM.

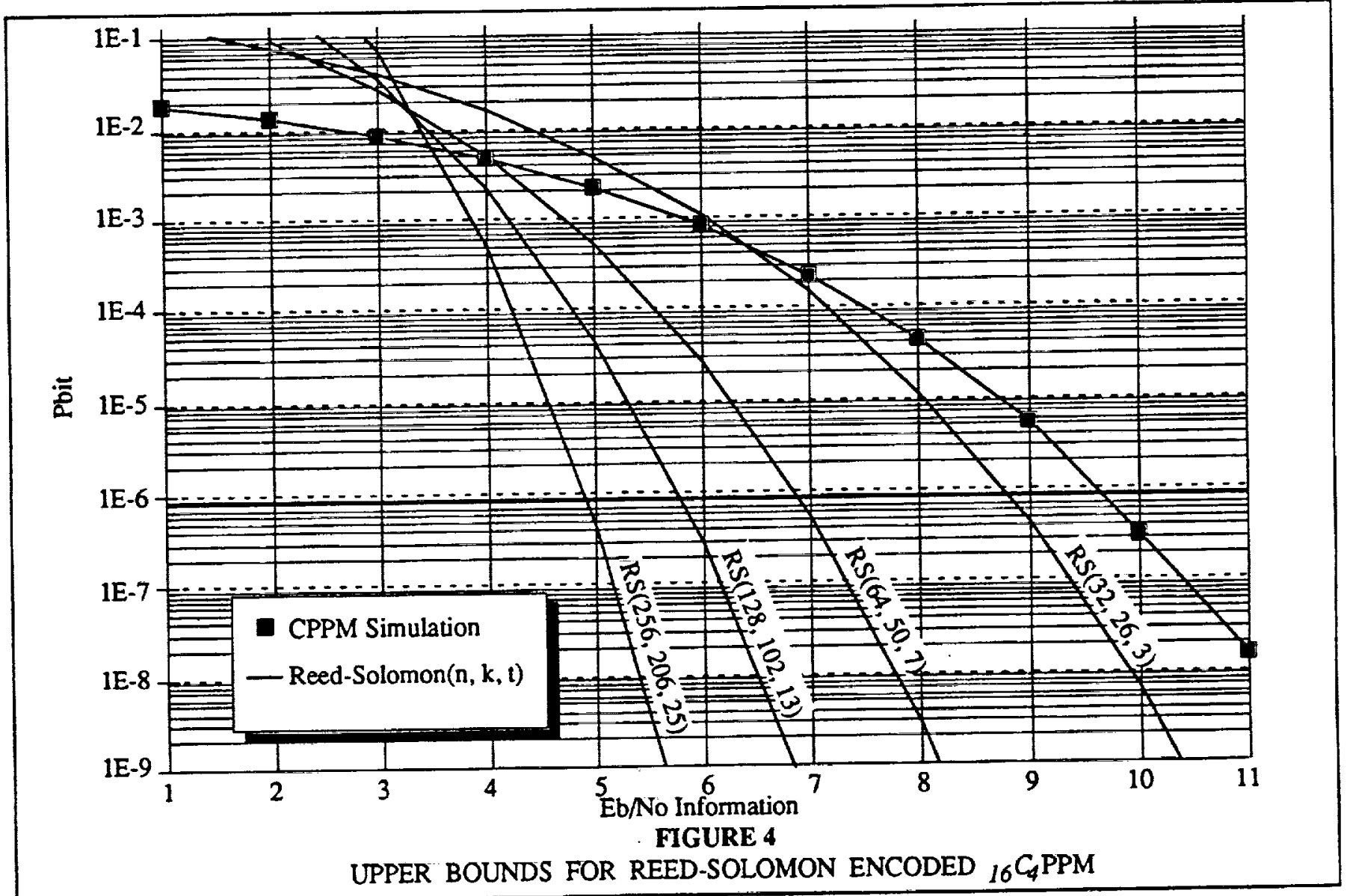




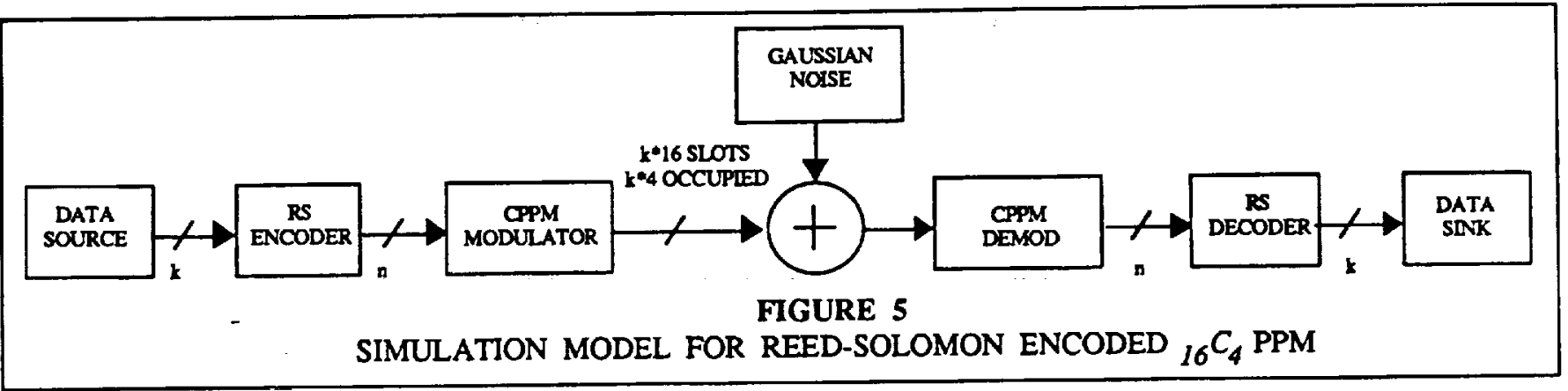

\section{IMPLEMENTATION APPROACH}

The primary reasons for choosing to investigate the specific case of ${ }_{16} C_{4} \mathrm{PPM}$ were its similarity to QPPM in terms of laser peak-to-average power and duty cycle requirements, and the minimal impact it places on the design and implementation of a conventional QPPM modem (modulator/demodulator). Under the High-speed Laser Integrated Terminal Electronics (HiLITE) Project, NASA is implementing a dual-channel 325 Mbps QPPM modem for potential use in intersatellite links ${ }^{6}$. A future objective of this investigation into CPPM is to modify the existing QPPM modem for ${ }_{16} C_{4}$ PPM operation. The salient functions of either modem implementation are illustrated in the block diagram (Figure 6).

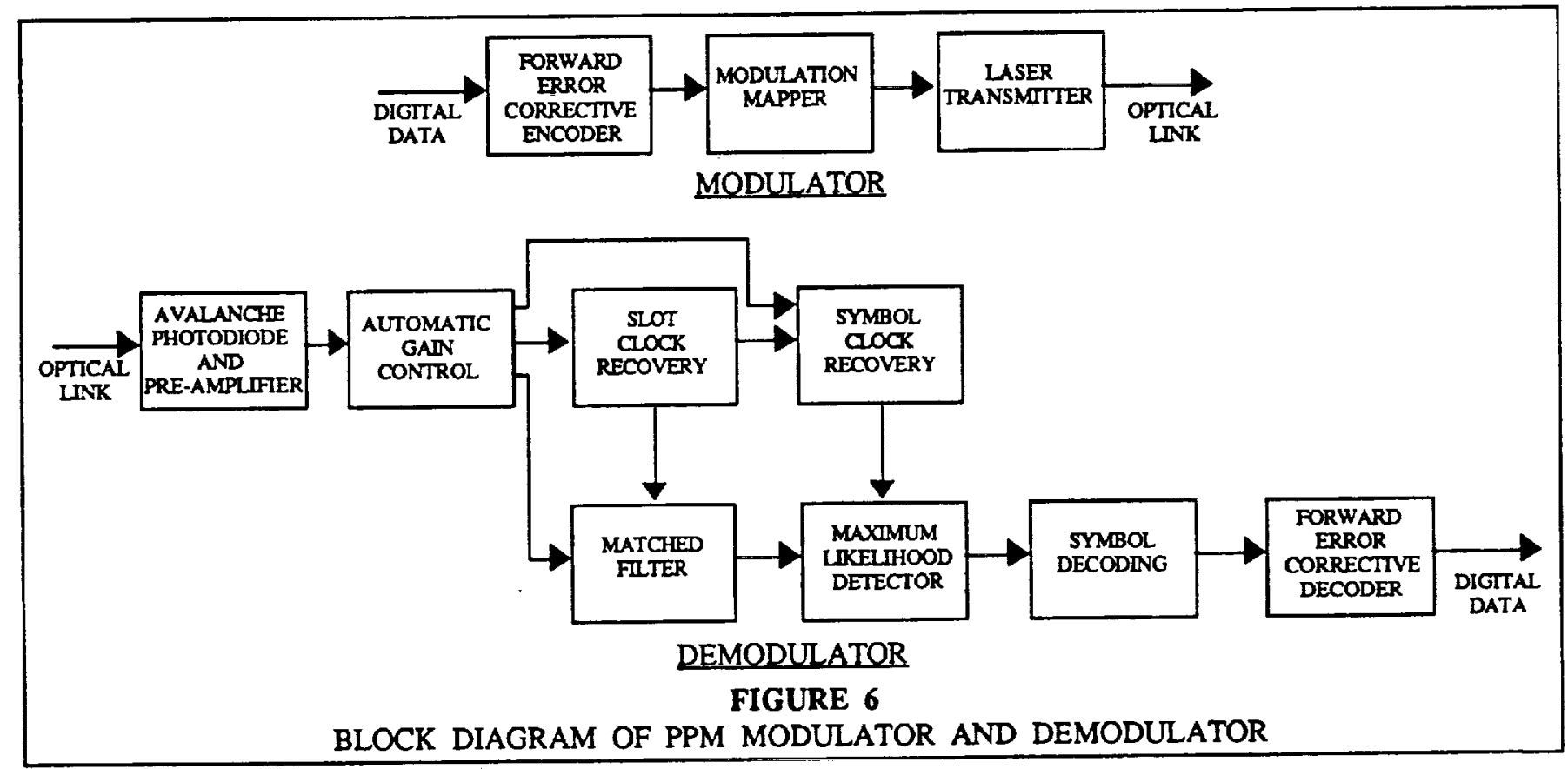

\subsection{Modulator}

All modulation functions are implemented in digital electronics. The FEC encoder takes an uncoded data stream and inserts redundant bits of information to improve the error detection and correction capability at the receiver. The encoded data stream emerges at a data rate higher than the incoming stream by a factor of the inverse of the code rate.

The only other function to be performed before modulating the laser is modulation mapping, a process that creates the appropriate transmitted symbols for a collection of bits in the data stream. In QPPM, the alphabet is very small (only 4 symbols) and the modulation mapping function is implemented in simple logic operating on two bits at a time. A look-up table is used to implement the ${ }_{16} C_{4} \mathrm{PPM}$ modulation mapping function. Bits of the encoded data stream are grouped ten at a time and then used to address a 16 kilobit mapping memory. The increase in data throughput available from CPPM is most easily recognized in the modulation mapper. Over a duration of sixteen slots, the QPPM modulator conveys eight bits of coded 
data in four sequential symbols, whereas ${ }_{16} C_{4} \mathrm{PPM}$ conveys ten bits in one longer symbol. In either case, the modulation mapper drives the laser and the optical pulse stream for the two modulation formats is nearly identical.

\subsection{Demodulator clock recovery}

In the demodulator, the signal recovered by an avalanche photodiode and pre-amplifier is further amplified and regulated by an automatic gain control (AGC) circuit. Following the AGC, clock and data recovery occur in parallel hardware paths. In the clock recovery path the slot clock is first acquired and synchronized to the incoming signal. The slot clock is later used to sample a matched filter in the data recovery path at the optimal instant. In the Hi-LITE QPPM demodulator two slot clock recovery techniques using commercial hardware are available for comparison: an injection-locked oscillator module; and single chip digital phase- locked loop. Since the ${ }_{16} C_{4}$ PPM modulated signal has nearly identical duty-cycle characteristics, the same slot clock recovery circuits can be used.

The symbol clock is derived directly from the slot clock by counting the number of slot clock cycles per symbol. The symbol clock is then synchronous with the slot clock. Aligning the symbol clock to the recovered waveform (symbol timing recovery) is not nearly so straightforward. In both $M$-ary PPM and CPPM, the symbol timing is recovered from a binary representation of the received waveform regulated in amplitude by the AGC circuit. Symbol timing recovery becomes more difficult as $M$ increases in $M$-ary PPM since there are fewer pulses available to firmly establish the symbol boundaries, and AGC is more difficult as well. Any symbol timing error in PPM produces a catastrophic error on the recovered data stream. Accordingly, simple, robust symbol timing recovery techniques are required for the power-efficient PPM techniques.

In QPPM, a simple threshold comparator in the demodulator is sufficient to create a rough approximation of the digital data steam used to modulate the laser in the transmitter. Then, some characteristic of the QPPM waveform is exploited to establish the symbol timing. One technique uses the periodic occurrence of back-to-back pulses on either side of a symbol boundary to drive a second phase-locked loop 16. In the Hi-LITE QPPM demodulator a robust digital technique is used to compare the four possible phases of the symbol clock and select the one that produces symbols containing one and only one pulse. Neither technique would offer an acceptable symbol timing error for the more power efficient M-ary PPM techniques such as 256-PPM.

CPPM offers a simple solution to the symbol timing recovery problem. As discussed previously, not all of the 1820 possible symbols in ${ }_{16} C_{4} \mathrm{PPM}$ are needed to convey 10 bits of information. If all symbols that have a pulse in one specific slot, the last slot in the symbol for example, are expurgated from the set, there are still ${ }_{15} C_{4}=1365$ available symbols from which to choose the final 1024. The absence of a pulse just before every symbol boundary can be exploited to recover symbol timing in either of two ways. As in QPPM demodulation, a second phase locked loop can be used. Here, the loop will lock on to the spike of energy at the symbol clock frequency. Alternatively, a fully digital technique can be used to step through the sixteen possible symbol clock phases until a slot with an arbitrarily few occurrences of a pulse (zero in the limit) is identified. Once the symbol timing is established, the timing acquisition circuit can be used to flag a potential symbol timing error and start a verification or reacquisition process.

\subsection{Demodulator dala recovery}

Data recovery consists of matched filtering, maximum likelihood detection, symbol decoding and FEC decoding. The matched filter used in the Hi-LITE QPPM demodulator is a commercial fifth order Bessel lowpass filter. This filter approximates a raised cosine filter and is an acceptably close complement to the sum of all distortions between the input to the laser diode and the output of the avalanche photodiode and pre-amplifier 17 . Because of its similarity to the characteristics of QPPM, the same matched filter can be used in ${ }_{16} C_{4}$ PPM. The matched filter accumulates a voliage representing the total energy from all the photons received in each slot period. The timing of the slot clock is adjusted during hardware assembly to insure that the matched filter is sampled as close as possible to the end of a slot period.

In PPM, the symbol clock is used to identify which slots belong to a given symbol. The peak voltage of each of the slots in a symbol can then be compared against each other to determine which slot most likely contained the pulse. Once the most likely slot is detected, either combinatorial logic or a memory lookup table is used to decode the detected symbol into binary data. If the binary data was FEC encoded at input to the modulator, then error detection and correction is performed at the output of demodulator according to the coding scheme employed.

While FEC decoding is readily performed in dense, high-speed digital logic, maximum likelihood detection may be more efficiently realized in analog circuitry at high speeds. This is not difficult for $M$-ary PPM when $M$ is small. For example, to compare the voltages representing the energy of all four slots in QPPM requires $M(M-I) / 2=6$ comparators. 
However, to compare even sixteen voltages as in 16-PPM would require 120 comparisons. A very different technique would be required for practical implementation of 256-PPM maximum likelihood detection. In addition, the bandwidth of the processing electronics for 256-PPM would be double that for QPPM due to the increased number of slots per fixed symbol duration. Conversely, the electronic bandwidth requirements for ${ }_{16} C_{4} \mathrm{PPM}$ are the same as for QPPM. The number of pulses per symbol increases from one to four, but the symbol duration increases by a factor of four as well. Although ${ }_{a} C_{b} \mathrm{PPM}$ in general requires significantly fewer comparisons than higher $M$-ary PPM, it also requires that the $b$ most likely slots be identified. A circuit called the winner-take-all (WTA) performs this operation in a readily expandable fashion.

\subsection{Winner-take-all circuit}

The WTA circuit functions as an $a$-input comparator. It compares a set of $a$ analog voltages against each other and produces a digital word output indicating which of the $a$ voltages was the largest. Thus, a WTA is a generalization of a twoinput comparator circuit. As such, it can be directly applied to $M$-ary PPM in place of an array of comparators. In CPPM, however, the detection of the $b$ slots with maximal energy out of a group of $a$ slots requires a different detection approach than that for one of $a$. The same number of voltage comparisons would be needed, but the logic driven by the comparators would become much more complex.

Although future improvements from shrinking device sizes will offset the increase in the number of components, circuit design methods such as those being developed for WTA circuits offer another method to detect permutation modulation schemes, with considerable reduction in component count. WTA circuits, by performing as a-input comparators, reduce the number of comparisons to essentially a single type of comparison. The WTA function automatically incorporates the logic gates that identify the winner, so that the wiring problem associated with using two input comparators and subsequent logic is drastically simplified. Finally, because WTA suructures can be compactly built on a single chip, high speed and large number of intemal connections simplifies the maximum likelihood detector design considerably.

In order to investigate the use of WTA circuits for CPPM and assess their advantages and disadvantages, two types of circuits were designed at Ohio State University and fabricated. The circuits were tested to determine critical performance parameters such as the minimum resolution between the inputs needed to achieve a winner, and criteria that limit the speed of the response. Two different circuits that can perform a one-out-of- five WTA function were designed and fabricated using the MOSIS 2um, p-well CMOS process. The die photograph is shown in Figure 7.

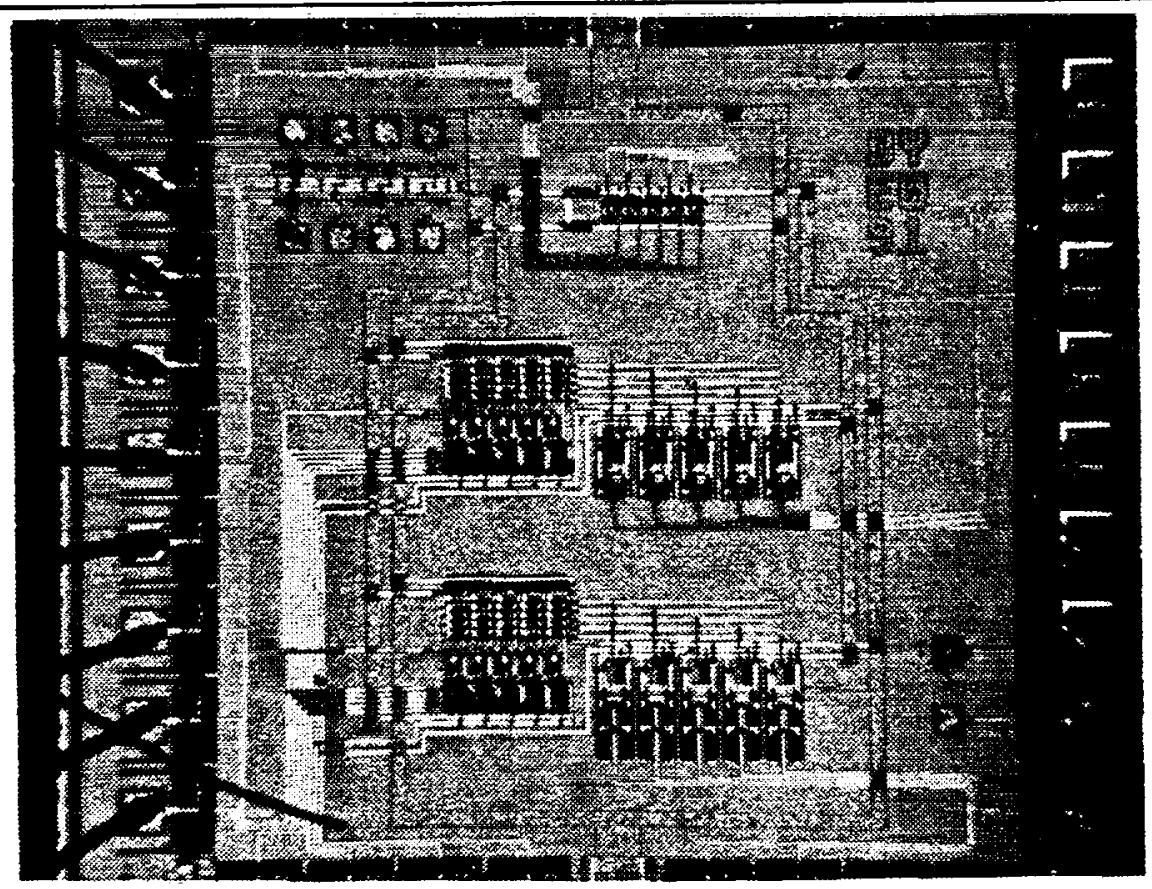

FIGURE 7

WINNER-TAKE-ALL DIE PHOTOGRAPH 
The two types of WTA circuits are referred to as: (1) Enabled NOR structure; and (2) Differential WTA. The Enabled NOR structure was approximately $0.4 \mathrm{~mm} \times 0.85 \mathrm{~mm}$ in die area, while the Differential WTA was approximately $0.34 \mathrm{~mm} x$ $0.19 \mathrm{~mm}$ in die area. Both types of circuits were operational, with the minimum resolution achieved being $1 \%$ of full scale. The Enabled NOR structure was shown to operate up to a $50 \mathrm{MHz}$ rate. A factor of ten increase is expected from implementation in a higher speed electronic process, allowing for conversion rates beyond $500 \mathrm{MHz}$.

The Enabled NOR structure operates as a fully interconnected set of NOR gates. For example, a one-out-of-four WTA is made up of four 3-input NOR structures, where each NOR drives the other three. A layout example of an Enabled NOR structure is shown in Figure 8. The analog voltages to be compared are brought in as currents, and are input where the VSS connection would normally be for a typical NOR gate. In an interconnected set of NOR gates, the final state will be that one and only one of the NOR outputs will be high. The differential WTA circuit is analogous to a differential pair, but instead of 2 input branches, there are $a$ input branches. The purpose of a differential pair in a comparator is to steer all current into one branch or the other from a common current sink. The a-input differential WTA circuit steers all the current from a common current sink into one of the $a$ branches. Due to considerable interest in the image processing community in the development of WTA circuits for image processing, new types of WTA circuits are continually being developed 18,19 .

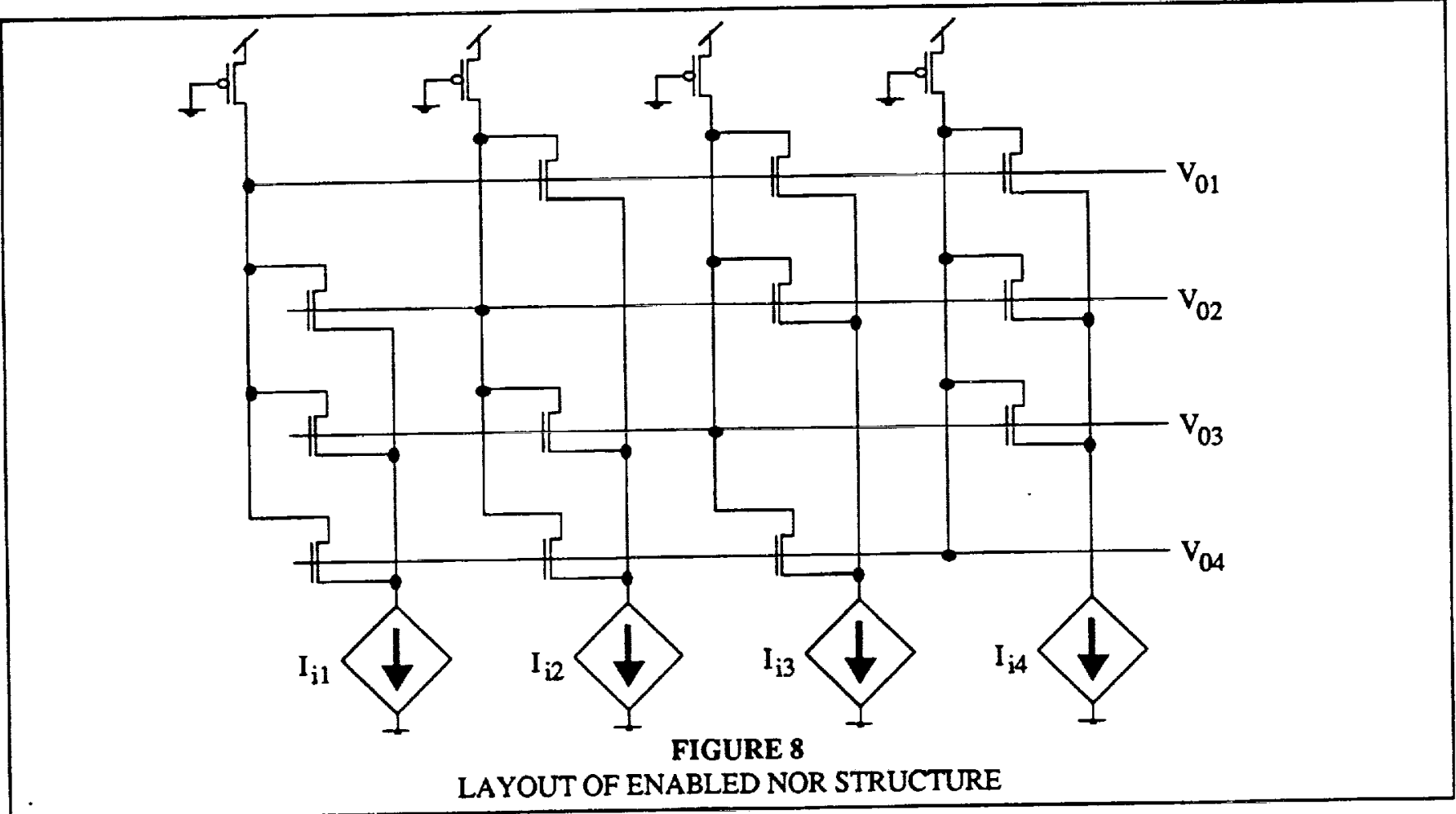

In order to develop a WTA circuit that can detect the highest voltage in $b$ out of $a$ slots, an enhanced version of the Enabled NOR structure is being developed for ${ }_{16} C_{4} \mathrm{PPM}$. The approach uses four interconnected WTA structures, each of which detects a winning slot and prevents the other three from detecting the same slot. The same inhibiting connection that is used within a one-out-of- $a$ WTA circuit is extended to prevent the succeeding WTA circuits from selecting the same slot. There are several methods to accomplish such inhibiting connections. As the first WTA circuit settles on a winning slot, the output corresponding to that slot is sent into the second circuit inhibiting the selection of the same winning slot. Likewise, the output wires from the first and second WTA circuits are routed to the third circuit to force the selection of the third highest slot and so on. This technique is readily expandable to more complex CPPM schemes.

\section{CONCLUDING REMARKS}

CPPM has been shown to be viable for use in modern direct-detection, free-space laser communication systems, including intersatellite links. The case of ${ }_{16} C_{4}$ PPM offers a $25 \%$ throughput improvement over QPPM that can be exchanged for at least a $4.7 \mathrm{~dB}$ improvement in power efficiency when a Reed-Solomon $(256,206,25)$ FEC code is employed. 
Conventional QPPM slot synchronization techniques were shown to be directly applicable to CPPM, while the proposed datato-symbol mapping design aids the ${ }_{16} C_{4}$ PPM demodulator in performing symbol synchronization. Maximum likelihood detection based on an analog WTA circuit was shown to be a practical realization approach to CPPM demodulation.

In general, CPPM can be optimized to match specific laser diode peak-to-average power and duty cycle characteristics. For example, ${ }_{12} C_{2}$ PPM could be selected for a diode with higher peak-to-average power capability and less stringent duty cycle requirements, both of which affect laser diode reliability. CPPM also reduces the required electronic bandwidth for high throughput systems. The BER performance of uncoded and FEC encoded CPPM needs to be investigated more thoroughly. Potential improvements may result from performing the modulation mapping prior to FEC coding, similar to conventional trellis coding of phase shift keyed radio frequency communications. In this case, gains could be realized due to an expanded signal set and soft-decision decoding. Finally, a faster WTA circuit should be developed to demonstrate the novel maximum likelihood detection technique within a high-speed CPPM demodulator.

\section{Z. REFERENCES}

1. M. Fitzmaurice, R. Bruno, "NASAJGSFC Program in Direct Detection Optical Communications for Intersatellite Links", in Optical space communication; Proceedings of the Meeting, Paris, France, April 26-29, 1989 ( A90-38001 16-17). Bellingham, WA, Society of Photo-Optical Instrumentation Engineers, 1989, pp. 10-23.

2. M. A. Krainak, "Optoelectronics Research for Communication Programs at the Goddard Space Flight Center", in MILCOM 91 - IEEE Military Communications Conference, McLean, VA, November 4-7, 1991, Conference Record, Vol. 3 (A9254751 23-32 ). New York, Institute of Electrical and Electronics Engineers, Inc., 1991, p. 1091-1097.

3. J. R. Lesh, "Deep Space Optical Communications - a Program Update", in Free-space laser communication technologies II; Proceedings of the Meeting, Los Angeles, CA, January 15-17, 1990 ( A91-22776 08-17). Bellingham, WA, Society of Photo-Optical Instrumentation Engineers, 1990, pp. 530-540.

4. F. M. Davidson, X. Sun, "Slot Clock Recovery in Optical PPM Communication Systems with Avalanche Photodiode Detectors", IEEE Trans. Comm., vol. 37, November 1989, pp. 1164-1172. Research sponsored by NASA.

5. Xiaoli Sun, Frederic M. Davidson, "Direct Detection Optical Intersatellite Link at $220 \mathrm{Mbps}$ using AlGaAs Laser Diode and Silicon APD with 4-ary PPM signalling", Interim Progress Report, September 1989 - February 1990, Johns Hopkins University, Baltimore, MD, Deparment of Electrical and Computer Engineering.

6. J. Budinger, S. Kerslake, L. Nagy, M. Shalkhauser, N. Soni, M. Cauley, J. Mohamed, R. Romanofsky, P. Lizanich, D. Mortensen, "Quaternary Pulse Position Modulation Electronics for Free-Space Laser Communications," NASA Technical Memorandum 104502, AIAA-91-3471, presented at AIAA Conference on Advanced Space Exploration Initiative Technologies, September 1991.

7. C. N. Georghiades, "Some Implications of TCM for Optical Direct-Detection Channels", IEEE Trans. Comm., vol. 37. May 1989, pp. $481-487$.

8. D. Slepian, "Permutation Modulation", Proc. IEEE, vol. 53, pp. 228-236, March 1965.

9. E. Brookner, "Nonorthogonal Coding", IEEE Trans. Communication Technology, vol. COM-13, No. 4, pp. 550-552, December 1965.

10. E. Brookner, "The Performance of FSK Permutation Modulations in Fading Channels and Their Comparison Based on a General Method for the Comparison of M-ary Modulations", IEEE Trans. Communication Technology, vol. COM-17, No. 6, pp. 616-640, December 1969.

11. H.L. Schneider, "Data Transmission with FSK Permulation Modulation", Bell Syst. Tech. J., vol. 47, pp. 1131-1138, July-August 1968. 
12. P.K. Wagner, J.M. Budinger, and M.J. Vanderaar, "Combinatorial FSK Modulation for Power-Efficient High-Rate Communications", NASA Technical Memorandum 105188, AIAA-91-3532.

13. B. Sklar, "Digital Communications Fundamentals and Applications", Prentice Hall, p. 254, 1988.

14. F. Davidson, X. Sun, "Gaussian Approximation versus Nearly Exact Performance Analysis of Optical Communication Systems with PPM Signalling and APD receivers", IEEE Trans. Comm., vol. 36, No. 11, pp. 1185-1192, November, 1988.

15. SPWTM - The DSP Framework ${ }^{\text {TM }}$, User's Guide and Tutorial, Comdisco Systems, Inc, 1992.

16. X. Sun, F. M. Davidson, "Word Timing Recovery in Direct Detection Optical PPM Communication Systems with Avalanche Photodiodes using a Phase-Locked Loop", IEEE Trans. Comm., vol. 38, May 1990, pp. 666-673. Research sponsored by NASA.

17. F. M. Davidson, X. Sun, "Reduced Electrical Bandwidth Receivers for Direct Detection 4-ary PPM Optical Communication Intersatellite Links", Interim Progress Report, April 1991 - October 1991, Johns Hopkins University, Baltimore, MD, Department of Electrical and Computer Engineering.

18. M. Hahowald and T. Delbruck, "Cooperative Stereo Matching Using Static and Dynamical Image Features," in Analog VLSI Implementations of Neural Systems, Kluwer Academic Publishers, 1989, pp. 213-232.

19. Y. Ni, F. Devos, and A. Legegue, "An Analog Smart Sensor for Active Obstacle Detection", to appear in the proceedings of the 1993 IEEE Circuits and Systems Conference. 
Public reporting burden for this collection of information is estimated to average 1 hour per response, including the time for reviewing instructions, searching existing data sources, Public reporting burdenion the collection of information. Send comments regarding this burden estimate or any other aspect of this gathering and Davis Highway, Suite 1204, Arlington, VA 22202-4302, and to the Ofice of Management and Budget, Paperwork Reduction Project (0704-0188), Washington, DC 20503.
1. AGENCY USE ONLY (Leave blank)
2. REPOAT DATE
January 1993
3. REPORT TYPE AND DATES COVEAED
Technical Memorandum

\section{TITLE AND SUBTIILE}

Combinatorial Pulse Position Modulation for Power-Efficient Free-Space Laser Communications

6. AUTHOR(S)

James M. Budinger, M. Vanderaar, P. Wagner, and Steven Bibyk

\section{PERFORMUNG ORGANIATION NAME(S) AND ADDRESS(ES)}

National Aeronautics and Space Administration

Lewis Research Center

Cleveland, Ohio 44135-3191

\section{SPONSORING/MONITORING AGENCY MAME(S) AND ADDRESS(ES)}

National Aeronautics and Space Administration

Washington, D.C. 20546-0001
WU-506-72-21

5. FUNDING NUMBERS

B PERFORUING ORGANRATION REPORT NUUBER

E-7961

10. SPONSORING/WONTTORING AGENCY REPORT NUMBER

NASA TM-106241

\section{SUPPLEMENTAAY NOTES}

Prepared for the Society of Photo-Optical Instrumentation Engineers sponsored by the International Society for Optical Engineering, Los Angeles, California, January 16-23, 1993. James M. Budinger, NASA Lewis Research Center; M. Vanderaar and P. Wagner, Sverdrup Technology, Inc., Lewis Research Center Group, 2001 Aerospace Parkway, Brook Park, Ohio 44142; and Steven Bibyk, Ohio State University, Columbus, Ohio 44321. Responsible person, James M. Budinger, (216) 433-3496.

12a. DISTRIBUTION/AVALABILTY STATEMENT

12b. DISTAIBUTION CODE

Unclassified - Unlimited

Subject Category 32

\section{ABSTRACT (Maximum 200 words)}

A new modulation technique called combinatorial pulse position modulation (CPPM) is presented as a power-efficient alternative to quaternary pulse position modulation (QPPM) for direct-detection, free-space laser communications. The special case of ${ }_{16} \mathrm{C}_{4} \mathrm{PPM}$ is compared to QPPM in terms of data throughput and bit error rate (BER) performance for similar laser power and pulse duty cycle requirements. The increased throughput from CPPM enables the use of forward error corrective (FEC) encoding for a net decrease in the amount of laser power required for a given data throughput compared to uncoded QPPM. A specific, practical case of coded CPPM is shown to reduce the amount of power required to transmit and receive a given data sequence by at least $4.7 \mathrm{~dB}$. Novel hardware techniques for maximum likelihood detection and symbol timing recovery are presented.

\section{SUBJECT TERMS}

Modulation; Pulse position; Laser communication; Bit error rate; Coding

\begin{tabular}{|c|c|c|}
\hline $\begin{array}{c}\text { 17. SECURTY CLASSIFICATION } \\
\text { OF REPORT } \\
\text { Unclassified }\end{array}$ & $\begin{array}{c}\text { 18. SECURTY CLASSIFICATION } \\
\text { OF THIS PAGE } \\
\text { Unclassified }\end{array}$ & $\begin{array}{c}\text { 19. SECURTY CLASSIFICATION } \\
\text { OF ABSTRACT } \\
\text { Unclassified }\end{array}$ \\
\hline
\end{tabular}


, - 